\title{
Surfactant-free Colloidal Particles with Specific Binding Affinity
}

Casper van der Wel, ${ }^{\dagger}$ (i) Nelli Bossert, ${ }^{\dagger}$ Quinten J. Mank, ${ }^{\dagger}$ Marcel G. T. Winter, ${ }^{\dagger}$ Doris Heinrich, ${ }^{\dagger,}$ and Daniela J. Kraft*, ${ }^{\dagger}$

${ }^{\dagger}$ Biological and Soft Matter Physics, Huygens-Kamerlingh Onnes Laboratory, Leiden University, P.O. Box 9504, 2300 RA Leiden, The Netherlands

${ }^{\ddagger}$ Fraunhofer Institute for Silicate Research, Neunerplatz 2, 97082 Würzburg, Germany

\section{Supporting Information}

ABSTRACT: Colloidal particles with specific binding affinity are essential for in vivo and in vitro biosensing, targeted drug delivery, and micrometer-scale self-assembly. Key to these techniques are surface functionalizations that provide high affinities to specific target molecules. For stabilization in physiological environments, current particle coating methods rely on adsorbed surfactants. However, spontaneous desorption of these surfactants typically has an undesirable influence on lipid membranes. To address this issue and create particles for targeting molecules in lipid membranes, we present here a surfactant-free coating method that combines high binding

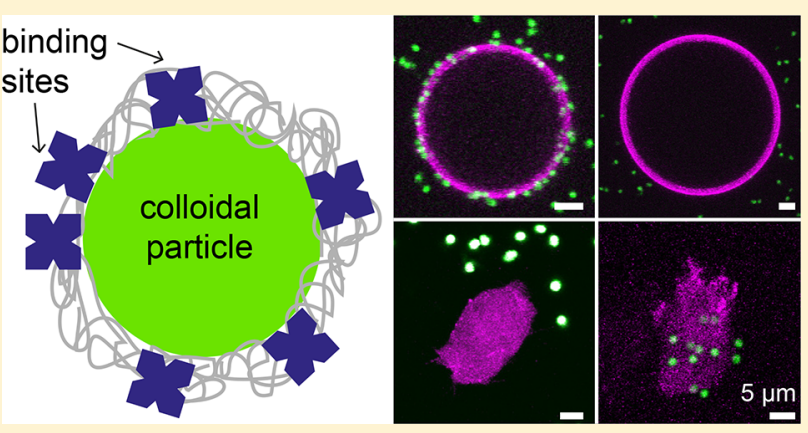
affinity with stability at physiological conditions. After activating charge-stabilized polystyrene microparticles with EDC/Sulfo-NHS, we first coat the particles with a specific protein and subsequently covalently attach a dense layer of poly(ethyelene) glycol. This polymer layer provides colloidal stability at physiological conditions as well as antiadhesive properties, while the protein coating provides the specific affinity to the targeted molecule. We show that NeutrAvidin-functionalized particles bind specifically to biotinylated membranes and that Concanavalin A-functionalized particles bind specifically to the glycocortex of Dictyostelium discoideum cells. The affinity of the particles changes with protein density, which can be tuned during the coating procedure. The generic and surfactant-free coating method reported here transfers the high affinity and specificity of a protein onto colloidal polystyrene microparticles.

\section{INTRODUCTION}

Surfaces with a high affinity to specific molecules are crucial in biological applications such as biosensing ${ }^{1,2}$ and drug targeting. ${ }^{3,4}$ Central to these applications is a particle coating that provides a high affinity and specificity to certain target molecules of interest. For example, colloidal particles can be functionalized with an affinity to particular proteins that are overexpressed on the cellular membrane of a tumor cell, ${ }^{5}$ which enables local drug release at or even in targeted cells. We focus here on polystyrene microparticles that especially target molecules embedded in lipid membranes. Such particles are required in fundamental membrane studies such as membrane tether pulling, ${ }^{6,7}$ membrane viscosity measurements, ${ }^{8-10}$ and the quantification of membrane-mediated forces. ${ }^{11-14}$ Furthermore, it has recently been shown that a specific linkage between particles and lipid membranes can be adopted for the self-assembly of particles into mobile structures such as colloidal clusters, strings, and dynamic networks. ${ }^{15-18}$

A controllable affinity and specificity of colloidal particles requires a careful design of the surface coating process. ${ }^{1,2,19-22}$ Next to moieties that provide the actual affinity, hydrophilic polymers are typically attached to the particle surface, which are necessary to prevent the undesirable aggregation of particles at physiological salt concentrations. These seemingly contra- dicting requirements, specifically attracting moieties next to generically repelling polymers, are usually met by adsorbing an amphiphilic polymer onto the colloidal particles before or during the coating process. These polymers provide steric stabilization against aggregation while the functional binding sites of the particles are still accessible. However, amphiphilic polymers are known to interact with lipid membranes. ${ }^{23-26}$ If these colloidal particles are used in lipid membrane studies, desorbing surfactants may disrupt the lipid membranes and compromise the accuracy of the corresponding measurements. Therefore, a surfactant-free coating is required for studies involving lipid membranes.

To meet this requirement, we have developed a two-step method yielding stable and specific polystyrene microparticles that can be applied to lipid membrane studies. First, we created colloidal particles with a high affinity through immobilization of proteins that specifically bind to the desired target molecules. $^{27-29}$ We focus mostly on the use of NeutrAvidin, as its affinity to biotin is used in many practical applications. This protocol can readily be used to prepare particles with other

Received: June 16, 2017

Revised: August 25, 2017

Published: August 28, 2017 


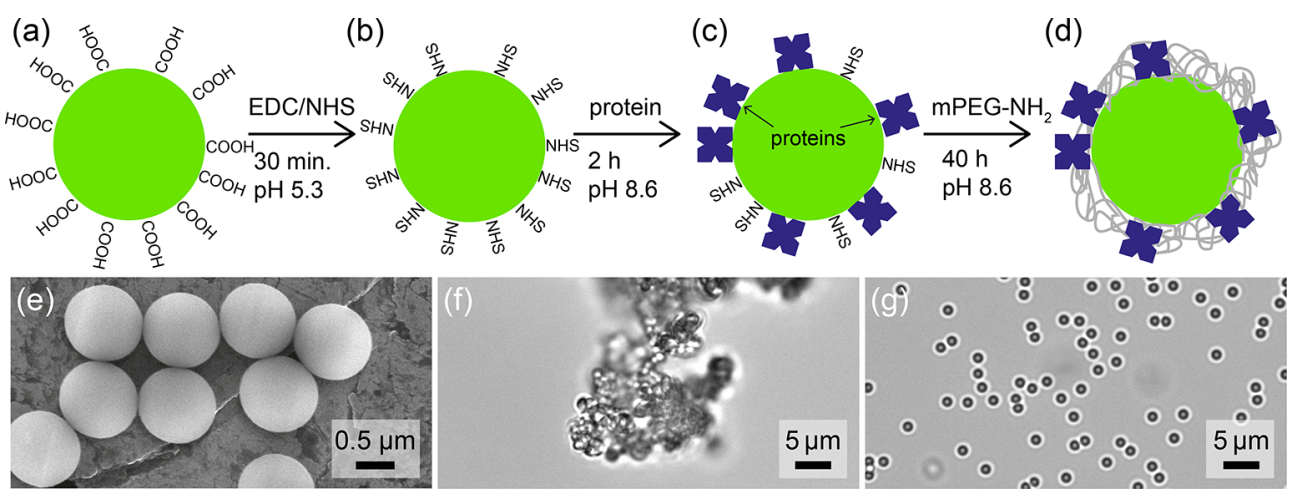

Figure 1. Illustration of the particle coating procedure. (a) Highly carboxylated (COOH) polystyrene particles were first activated using EDC/NHS so that the carboxylic acid groups form NHS esters. (b) The activated particles were then mixed with a precise quantity of protein at $\mathrm{pH}$ 8.6. (c) After a delay time of typically $2 \mathrm{~h}$, an excess of amine-functionalized methoxypoly (ethelyne) glycol ( $\mathrm{mPEG}-\mathrm{NH}_{2}$ ) was added to stabilize the particles. (d) The resulting particle coating contains the added proteins as well as a dense mPEG layer that provides colloidal stability. (e) A scanning electron micrograph of the uncoated particles. Light microscopy images after $1 \mathrm{~h} \mathrm{in} 1 \mathrm{M} \mathrm{NaCl}$ show that (f) the uncoated polystyrene particles aggregated strongly and $(\mathrm{g})$ the $\mathrm{mPEG}$-coated particles remained dispersed.

target specificity, as we demonstrate by also employing the sugar-binding protein Concanavalin A. Second, we do not stabilize the colloidal particles by using adsorbed surfactants but instead by covalent grafting of methoxylpoly(ethylene) glycol (mPEG) to the particle surface in such a way that it preserves the affinity and specificity of the tested proteins. At sufficiently high surface densities, mPEG is known to suppress particle aggregation. ${ }^{30}$ Furthermore, a dense $\mathrm{mPEG}$ coating has been proven to suppress nonspecific protein adsorption on the particles, thus providing "stealth" properties necessary for drug targeting in living organisms. ${ }^{1,3}$ These properties make mPEG an ideal choice as a stabilizer for colloidal particles at physiological conditions.

In this article, we will first describe the synthesis method and study the effect of various parameters on the resulting surface density of avidin. Then, we will assess the binding affinity of avidin-functionalized colloidal particles on biotinylated giant unilamellar vesicles (GUVs). Finally, we will show that avidinfunctionalized particles bind specifically to biotinylated lipid membranes and Concanavalin A-functionalized particles to the cellular membrane of Dictyostelium discoideum cells.

\section{EXPERIMENTAL SECTION}

Materials. N-Hydroxysulfosuccinimide sodium salt (98\%, SulfoNHS), sodium phosphate $\left(99 \% \mathrm{Na}_{2} \mathrm{HPO}_{4}\right)$, Pluronic F-127, and hydrochloric acid $(\mathrm{HCl})$ were acquired from Sigma-Aldrich. Methoxypoly(ethylene) glycol amine (mPEG, $\left.M_{\mathrm{w}}=5000\right)$ was acquired from Alfa Aesar. Sodium hydroxide $(\mathrm{NaOH}, 98.5 \%)$, sodium chloride $(\mathrm{NaCl}, 99 \%)$, and sodium azide $\left(\mathrm{NaN}_{3}, 99 \%\right)$ were acquired from Acros Organics. 1-Ethyl-3-(3-dimethylaminopropyl) carbodiimide hydrochloride (99\%, EDC) was acquired from Carl Roth. NeutrAvidin (avidin) and biotin-4-fluorescein were acquired from Molecular Probes. Biotin-5'-TTTAATATTA-3'-Cy3 DNA oligonucleotides (btn-DNA-Cy3) were acquired from Integrated DNA Technologies. $\Delta 9$-Cis 1,2-dioleoyl-sn-glycero-3-phosphocholine (DOPC), 1,2-dioleoyl-sn-glycero-3-phosphoethanolamine- $\mathrm{N}$-[biotinyl(polyethylene glycol)2000] (DOPE-PEG-biotin), 1,2-dioleoyl-sn-glycero-3-phosphoethanolamine- $N$-[methoxy(polyethylene glycol)2000] (DOPE-PEG), and 1,2-dioleoyl-sn-glycero-3-phosphoethanolamine$\mathrm{N}$-(lissaminerhodamine B sulfonyl) (DOPE-rhodamine) were acquired from Avanti Polar Lipids. Trypsin was acquired from Life Technologies. All chemicals were used as received. Deionized water with $18.2 \mathrm{M} \Omega \mathrm{cm}$ resistivity was used, which was obtained using a Millipore Filtration System (Milli-Q Gradient A10). PBS consisted of $12.5 \mathrm{mM} \mathrm{Na}_{2} \mathrm{HPO}_{4}, 50 \mathrm{mM} \mathrm{NaCl}$, and $3 \mathrm{mM} \mathrm{NaN}_{3}$ with a $\mathrm{pH}$ of 7.5 .
Phosphate buffer for cell culture consisted of $2.00 \mathrm{~g} / \mathrm{L}$ of $\mathrm{KH}_{2} \mathrm{PO}_{4}$ and $0.36 \mathrm{~g} / \mathrm{L}$ of $\mathrm{Na}_{2} \mathrm{HPO}_{4} \cdot \mathrm{H}_{2} \mathrm{O}$ with a $\mathrm{pH}$ of 6.0 . See the Supporting Infromation for the compositions of the HL5, 3T3, and A549 media.

Particle Preparation. Polystyrene microparticles were prepared using a surfactant-free dispersion polymerization protocol. ${ }^{31}$ The nonfluorescent particles employed in this article were $1.06 \pm 0.02 \mu \mathrm{m}$ in diameter, and the green fluorescent particles $\backslash 0.98 \pm 0.03 \mu \mathrm{m}$. Their $\zeta$-potentials in water ( $\mathrm{pH}$ 5.7) were determined to be $-54 \pm 4$ and $-55 \pm 4 \mathrm{mV}$, respectively. These strongly negative values reflect the high density of carboxylic acid groups $\left(1-10 \mathrm{~nm}^{-2}\right)$ on the particle surface. ${ }^{31}$ See the Supporting Information for the synthesis details.

Coating and Stabilization. The particle coating procedure was extended from a covalent $\mathrm{mPEG}$ grafting protocol from the literature ${ }^{30}$ (see Figure 1). All reactions were performed on a vortex mixer at $4{ }^{\circ} \mathrm{C}$. The polystyrene particles with carboxylic acid groups were activated by adding $8 \mathrm{mM}$ EDC and $2.5 \mathrm{mM}$ Sulfo-NHS to the particles, which were suspended at $20 \mathrm{~g} / \mathrm{L}$. The EDC and Sulfo-NHS were dissolved immediately before use. At this concentration, the hydrochloric acid in the EDC ensures a pH of 5.3, which was confirmed using a calibrated Hach PH17-SS ISFET $\mathrm{pH}$ probe. After $30 \mathrm{~min}$ of mixing, the $\mathrm{pH}$ was brought to 8.6 using $0.2 \mathrm{M} \mathrm{NaOH}$. At this $\mathrm{pH}$, NHS hydrolysis starts, which we suppressed by performing the reactions at $4{ }^{\circ} \mathrm{C}$. Then, 5-500 $\mu \mathrm{g}$ of protein (NeutrAvidin or Concanavalin A) was added to $750 \mu \mathrm{L}(15 \mathrm{mg})$ of the activated particles. After a delay time of $2 \mathrm{~h}, 4.0$ mg of mPEG5000- $\mathrm{NH}_{2}$ dissolved in $200 \mu \mathrm{L}$ of water was added. The reaction was allowed to proceed for at least $40 \mathrm{~h}$. For the remaining NHS groups to be removed, the $\mathrm{pH}$ was adjusted to 12 for 5 min using $1 \mathrm{M} \mathrm{NaOH}$. For some proteins, this high $\mathrm{pH}$ is undesirable, in which case $\mathrm{pH} 9$ may be used for a longer time period to hydrolyze the NHS esters at room temperature. ${ }^{32}$ The particles were washed one time with $10 \mathrm{mM} \mathrm{HCl}$ to neutralize the $\mathrm{pH}$ and three times with water. Finally, sodium azide was added to a concentration of $3 \mathrm{mM}$ to prevent bacterial growth. The colloidal stability of each sample was assessed as follows: $50 \mu \mathrm{g}$ of particles was dispersed in $10 \mu \mathrm{L}$ of $1 \mathrm{M} \mathrm{NaCl}$. After 1 $\mathrm{h}$ of mixing, the sample was diluted 20 -fold with $1 \mathrm{M} \mathrm{NaCl}$ and imaged using bright field microscopy (Figure $1 \mathrm{~g}$ ). The resulting particle suspensions can be stored for at least two months at $4{ }^{\circ} \mathrm{C}$. For longer shelf life, the particle suspensions should be frozen at $-20{ }^{\circ} \mathrm{C}$.

Avidin Titration Assay. The number of biotin binding sites on the particles was measured using a titration assay based on the binding of biotin-4-fluorescein to the particle-attached avidin. Because of the strong scattering of the particles, we could not measure the fluorescence of the avidin-bound biotin-4-fluorescein directly as described elsewhere. ${ }^{33}$ Instead, we determined the remaining fluorescence of the supernatant for different amounts of added biotin-4-fluorescein. From a single particle batch, precisely known aliquots of $0.3-0.6 \mathrm{mg}$ of particles were dispersed into $1.00 \mathrm{~mL}$ of PBS. Subsequently, quantities of $0-10 \mu \mathrm{L}$ of $1.8-7.3 \mu \mathrm{M}$ biotin-4- 
fluorescein were incubated with the particles for $20 \mathrm{~min}$ at $55^{\circ} \mathrm{C}$. After this, the particles were removed by centrifugation. Then, $750 \mu \mathrm{L}$ of supernatant was diluted with $750 \mu \mathrm{L}$ of PBS, and the fluorescence of each aliquot was determined using a Varian Cary Eclipse fluorescence spectrophotometer (ex. 494 nm, em. $524 \mathrm{~nm}$ ). From the intersection of the baseline fluorescence and the final slope of the titration curve, the amount of biotin binding sites in the sample was determined (see Figure S1). Together with the separately determined dry weight and diameter of the particles, the amount of biotin binding sites per particle was computed.

Avidin Fluorescence Assay. To measure the distribution of biotin binding sites on avidin-functionalized particles within a single batch, we added a fluorescent marker for use in fluorescence microscopy. The employed fluorescent marker was a biotin- and Cy3-functionalized DNA oligonucleotide, as DNA is well soluble in water and shows no aspecific adsorption to the particle surface. To stain the particle-attached avidin, we incubated $50 \mu \mathrm{g}$ of particles with $60 \mathrm{pmol}$ btn-DNA-Cy3 in $310 \mu \mathrm{L}$ of PBS buffer for $30 \mathrm{~min}$ at $55^{\circ} \mathrm{C}$ in a nuclease-free plastic microtube. To be able to use the same protocol for unstable particles, we added $0.5 \mathrm{wt} \%$ of the steric stabilizer Pluronic F-127 to the PBS buffer (see Materials for the contents). After cooling to room temperature, the samples were washed three times with water and one time with PBS. Finally, the samples were inserted into a rectangular capillary and imaged on a Nikon Ti-E microscope equipped with a Nikon 100× CFI Apo TIRF oilimmersion objective (NA = 1.49), Intensilight fluorescent lamp (ex. $540 \pm 25 \mathrm{~nm}$, em. $605 \pm 55 \mathrm{~nm}$ ), and a DS-Qil monochrome CCD camera with an exposure time that was set such that all particles had a fluorescence signal between the camera background and saturation levels $(100-800 \mathrm{~ms})$. In a separate experiment, the backgroundcorrected emission intensity per feature was confirmed to be proportional to the exposure time (see Figure S2). The resulting images were ensured to be in the same focal plane, and the integrated intensity per particle was computed using Trackpy. ${ }^{34}$

Sample Preparation. Giant unilamellar vesicles (GUVs) of 10$100 \mu \mathrm{m}$ diameter were prepared using the electroformation method. ${ }^{35}$ The composition was $97.5 \mathrm{wt} \%$ DOPC, $0.5 \mathrm{wt} \%$ DOPE-rhodamine, and either 2 wt \% DOPE-PEG-biotin or 2 wt \% DOPE-PEG. GUV samples were prepared by consecutive addition of $50 \mu \mathrm{L}$ of PBS (diluted to a total osmotic content of $100 \mathrm{mM}$ ), $0.5 \mu \mathrm{L}$ of $3 \mathrm{wt} \%$ particles, and $2 \mu \mathrm{L}$ of GUVs. Dictyostelium discoideum SadA-GFP LimRFP cells were cultured in HL5 medium. These imaging samples were prepared by subsequent addition of $50 \mu \mathrm{L}$ of phosphate buffer, $0.5 \mu \mathrm{L}$ of $1.5 \mathrm{wt} \%$ particles, and $10 \mu \mathrm{L}$ of cells.

All samples were prepared on a hydrophobic coverslip that was incubated for $15 \mathrm{~min}$ in a 5\% Pluronic F-127 solution and washed three times with the appropriate buffer. Images were taken 30-45 min after mixing. See the Supporting Information for additional details on the GUV preparation, cell culture, and imaging.

\section{RESULTS AND DISCUSSION}

We describe here a surfactant-free preparation method of colloidal particles that specifically bind to target molecules. In this method, polystyrene microparticles are first coated with a protein that provides the specific affinity and subsequently with a layer of $\mathrm{MPEG}$ that provides stability against aggregation under physiological conditions (see Figure 1). As protein we primarily use avidin, which has a high affinity to biotin, and further show that the method is readily transferable to the sugar-binding Concanavalin A. In the following, we will characterize the particle coating and show its affinity and specificity on vesicles as well as on living cells. First, we study the colloidal stability of the particles at various physiological conditions. Second, we quantify how the number of biotin binding sites on the avidin-coated particles depends on the synthesis parameters. Then, we measure the binding affinity of the avidin-coated particles to biotinylated membranes. Finally, we demonstrate that avidin-coated particles bind specifically to biotinylated membranes and that Concanavalin A-coated particles bind specifically to the glycocortex of $D$. discoideum cells.

Colloidal Stability. One of the most important requirements of a colloidal dispersion is its stability. Without a polymer coating, colloidal stability relies on Coulomb repulsion: the surface charge of the particles prevents aggregation in deionized water. At a physiological salt concentration, however, the Coulomb force is screened by counterions so that the short-ranged van der Waals force induces permanent particle aggregation (see Figure 1e). Therefore, steric stabilization is necessary to use colloidal particles at physiological conditions. This involves adsorbing or grafting a layer of well-soluble polymers to the particle surface. When particles approach each other, the layers on neighboring particles will interpenetrate, which costs free energy as the polymers can occupy less volume. ${ }^{36}$ For a sufficiently dense and thick polymer layer, this yields a repulsive force that counteracts the van der Waals attraction and protects the particles from aggregating at arbitrary salt concentrations. ${ }^{37}$

We achieve this steric stabilization here by covalently grafting methoxypoly(ethylene) glycol (mPEG5000) to the particles by an NHS/Sulfo-NHS linking procedure. ${ }^{30} \mathrm{mPEG}$ is an excellent candidate for stabilizing colloidal particles as it is uncharged and hydrophilic. Furthermore, mPEG coatings have been shown to reduce the nonspecific adhesion of particles to proteins, cells, and tissues. ${ }^{20}$ As these properties make the particles less visible to the immune system, mPEG coatings are particularly interesting for drug delivery applications. ${ }^{3}$

Indeed, we found that after $1 \mathrm{~h}$ in $1 \mathrm{M} \mathrm{NaCl}$, the mPEG5000-coated particles employed here showed excellent stability against aggregation. We observed that performing the mPEG coating at $4{ }^{\circ} \mathrm{C}$ for a minimum of $24 \mathrm{~h}$ is essential for obtaining the required colloidal stability, which is in agreement with earlier reports on mPEG-coated particles. ${ }^{30}$ The $\zeta$ potential before particle coating $(-55 \pm 4 \mathrm{mV})$ clearly becomes less negative after the mPEG coating (see Table 1), which provides additional evidence for the presence of a dense PEG layer. ${ }^{30}$ The remaining negative charge is caused by unreacted carboxylic acid groups.

\section{Table 1. $\zeta$-Potential of mPEG-Coated Particles ${ }^{a}$}

$\begin{array}{ll}\text { solvent } & \mathrm{p} \\ \text { water } & 5.7 \\ \text { PBS } & 7.3 \\ \text { HL5 } & 6.6 \\ \text { A549 } & 7.6 \\ \text { 3T3 } & 8.1\end{array}$

$\begin{array}{cc}\mathrm{pH} & \zeta \text {-potential }[\mathrm{mV}] \\ 5.7 & -24 \pm 4 \\ 7.3 & -18 \pm 4 \\ 6.6 & -15 \pm 5 \\ 7.6 & -7 \pm 5 \\ 8.1 & -7 \pm 5\end{array}$

${ }^{a}$ Measurements were taken after $1 \mathrm{~h}$ incubation in water, phosphate buffered saline (PBS), the D. discoideum medium HL5, and mammalian cell media A549 and 3T3. See Supporting Information for the precise contents of these media.

Furthermore, we assessed the stability of the mPEG-coated particles in a standard PBS buffer and in three typical cell culture media. After $1 \mathrm{~h}$ of mixing, we did not observe any aggregation of the particles inside any of these solvents. In HL5 medium, the $\zeta$-potential was similar to that in the PBS buffer, whereas we observed a significant change in both mammalian cell media (see Table 1). Although HL5 contains mostly salts, sugars, and amino acids, the A549 and 3T3 cell media contain significant amounts of proteins from bovine serum. Protein 
adsorption has been shown to be suppressed but not completely prevented by a dense mPEG coating such as that employed here. ${ }^{38,39}$ Therefore, we attribute the less negative $\zeta$ potential in the A549 and 3T3 cell media to nonspecific protein adsorption.

To summarize, the particles described here with a dense layer of $\mathrm{mPEG}$ on their surface do not show aggregation at a high salt concentration or in several cell culture media. To now provide these particles with an affinity to a specific molecule, we introduce an additional step in the procedure: we first coat the EDC/Sulfo-NHS-activated particles with a functional protein before saturating the surface with an excess of mPEG (see Figure 1). The resulting particles possess functional proteins as well as mPEG polymers on their surface. We still observed that these particles were stable in $1 \mathrm{M} \mathrm{NaCl}$ after $1 \mathrm{~h}$ for protein densities of up to $67 \mu \mathrm{g} / \mathrm{mg}$ of particles. Although the proteins occupy significant space on the particles (see next section), we conclude that the density and size of mPEG5000 is still sufficiently high to protect the particles from aggregating. This combination of covalent colloidal stability with a specific binding protein is key to targeting specific molecules on lipid membranes.

Accessible Binding Sites. In general, a specific binding affinity of a colloidal particle is provided by a finite number of binding sites on the particle surface. The number of sites will directly influence the binding affinity of the particle, and therefore, we quantified this parameter for the particles described here. During the coating, we immobilized the protein via NHS groups on the particles, which are known to form a covalent amine bond with random lysine residues that are typically present in the protein exterior. ${ }^{27,28}$ As an example, we coated particles with avidin, which is a protein that binds to biotin with high affinity $\left(\sim 17 k_{\mathrm{B}} T\right.$ per bond $\left.{ }^{40}\right)$. Because of this high affinity and the versatility of avidin, it has been widely applied as a connector in biochemistry, for instance as a binding site for attaching biotinylated monocolonal antibodies. ${ }^{41,42}$ We use NeutrAvidin here, which is a neutral and nonglycosylated avidin, and quantify the number of biotin binding sites using a combined titration and fluorescence assay for several synthesis conditions.

To rule out noncovalent adsorption of the avidin or mPEG on the particles during synthesis, ${ }^{43}$ we performed the protocol without the EDC/NHS activation (Figure 1a, b). The absence of NHS esters resulted in particles without biotin binding sites; at the same time, the particles were unstable in $1 \mathrm{M} \mathrm{NaCl}$. This proves that the NHS activation is necessary for avidin and mPEG binding. We conclude that both avidin and MPEG are covalently linked to particles via amine bonds through the EDC/NHS chemistry.

The number of biotin binding sites on the particles is controlled by the amount of avidin that is added during synthesis. To probe this relation, we changed the quantity of added avidin between 0 and $7 \mu \mathrm{g} / \mathrm{mg}$ of particles and measured the resulting average number of biotin binding sites per particle with a titration assay (Figure $2 \mathrm{a}$ ). Clearly, the amount of added avidin allows for continuously changing the average number of biotin binding sites per particle up to at least $2.9 \times 10^{4}$. Assuming a protein size of $5 \mathrm{~nm}$, we estimate that at this value approximately $1 \%$ of the particle surface is covered with avidin. The slope of the linear regression in Figure 2a provides information on the synthesis yield: the biotin binding capacity corresponds to 0.71 accessible biotin binding sites per added avidin protein, which has a total of 4 binding sites. Apart from
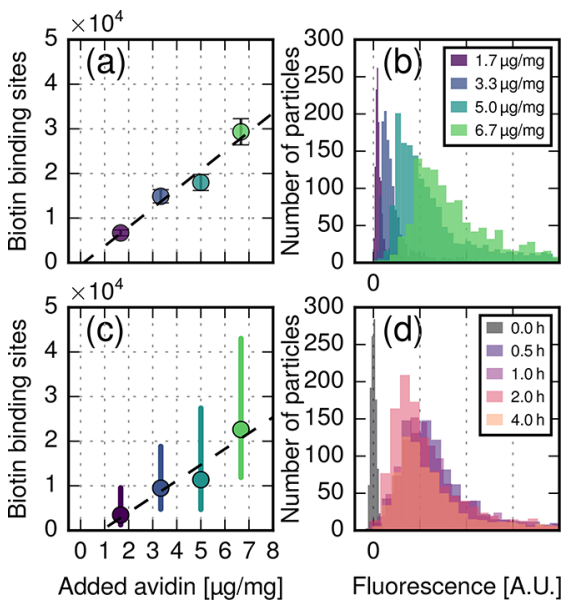

Figure 2. Amount of biotin binding sites on avidin-coated particles. (a) The average number of binding sites per particle measured by titration. Four particle batches with varying amounts of avidin were analyzed. Error bars denote the measurement precision. (b) Histograms of the btn-DNA-Cy3 fluorescence of the same particle batches (see legend) show a skewed distribution for all samples. Combining the average number of binding sites from the titration assay (a) with the distributions from the fluorescence microscopy assay (b), the distribution of binding sites within particles of a single batch could be recovered, which is displayed in (c). Here, error bars denote one standard deviation spread of biotin binding sites per particle in a single batch, and the disks mark the most frequently occurring value. Lines in (a) and (c) are linear regressions. (d) Histograms of the btn-DNA-Cy3 fluorescence of five particle batches with varying delay times between avidin and $\mathrm{mPEG}$ addition (see legend) at a fixed avidin concentration of $3.3 \mu \mathrm{g} / \mathrm{mg}$. As the distributions did not change after $0.5 \mathrm{~h}$, we concluded that the avidin coating was completed within $0.5 \mathrm{~h}$ after avidin addition.

the finite chemical yield of the avidin immobilization, we presume that some of the biotin binding sites are actually present on the particles but not accessible because of the random orientations of the avidin proteins, ${ }^{27}$ steric hindrance by the covalently grafted mPEG polymers, or unfolding of the proteins during the $\mathrm{pH} 12$ synthesis step.

Additionally, we measured the distribution of the number of biotin binding sites per particle using quantitative fluorescence microscopy. By incubating the particles with btn-DNA-Cy3, we visualized the accessible biotin binding sites for individual particles. As can be seen in Figure $2 b$, the resulting distributions are broad and skewed toward lower values of fluorescence. The relatively large spread is a consequence of the random immobilization of the avidin on the particle surfaces. For this effect to be mitigated, it is important that the added protein is well mixed with the particles during the coating procedure. Here, we achieved this by vortexing the mixture directly after the addition of protein to the particles.

By combining the distributions from the fluorescence assay with the quantitative results from the titration assay, we obtained the most frequently occurring number of biotin binding sites per avidin-coated particle, which is shown in Figure $2 c$ together with the one standard deviation spread. Because of the skewed distributions, the average number of biotin binding sites (Figure 2a) is higher than the most frequently occurring number of biotin binding sites (Figure 2c). Still, this quantity is linear with the amount of added avidin and therefore can be readily tuned by varying the avidin concentration. 

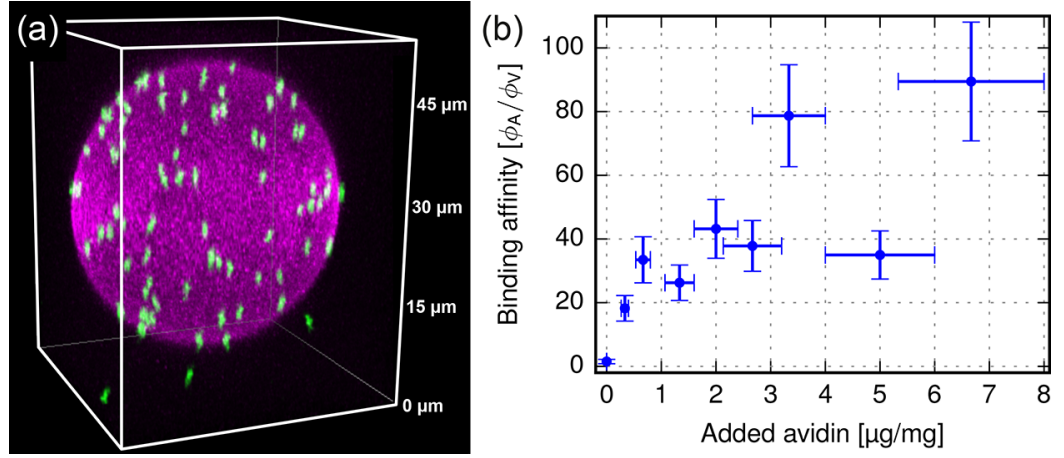

Figure 3. Binding affinity of avidin-coated particles on biotinylated lipid membranes. (a) Three-dimensional confocal image showing a biotinylated GUV (magenta) that has been incubated for $30 \mathrm{~min}$ with avidin-coated particles (green). (b) Membrane area coverage $\phi_{A}$ was measured and averaged over 10 randomly selected GUVs for varying amounts of avidin on the particles. The particle volume fraction $\phi_{\mathrm{V}}$ was $0.021 \pm 0.004$ vol $\%$. The vertical error bars denote the uncertainty in binding affinity due to the imprecision in particle volume fraction; the horizontal error bars denote the uncertainty in the amount of added avidin. The confocal image in (a) corresponds to the point at $3.3 \mu \mathrm{g} / \mathrm{mg}$ of avidin.

After activating the carboxylic acid particles with NHS, avidin and $\mathrm{mPEG}$ are added sequentially. As the two reactions compete with one another for the NHS esters, we used a delay time between avidin and mPEG addition so that the avidin immobilization is allowed to complete before mPEG starts to occupy the particle surface area. The duration of this delay may affect the final avidin density on the particles. To find the minimum delay time between the addition of avidin and mPEG, we varied the delay between 0 and $4 \mathrm{~h}$ at a fixed amount of avidin of $3.3 \mu \mathrm{g} / \mathrm{mg}$ of particles. See Figure $2 \mathrm{~d}$ for the corresponding distributions of the btn-DNA-Cy3 fluorescence. As the distributions do not change significantly between 0.5 and $4 \mathrm{~h}$, we conclude that the distribution of linker densities already reached a steady state within the first $0.5 \mathrm{~h}$. Throughout this article, we fixed the delay time at $2 \mathrm{~h}$ to ensure the complete grafting of proteins.

To summarize, we have shown that the reported particle coating method allows for tuning the average number of accessible biotin binding sites up to $2.9 \times 10^{4}$ per particle, which is sufficient to achieve high binding affinity and specificity, as will be demonstrated in the next sections.

Binding Affinity. In the previous section, we have shown that the avidin-coated particles bind fluorescently labeled biotin molecules. Next, we investigated whether this binding affinity also holds for lipid membranes that feature biotin target groups. As a model membrane, we used giant unilamellar vesicles (10$50 \mu \mathrm{m}$ in diameter) that contain biotinylated lipids. After 30 min incubation of these vesicles in a 0.02 vol \% particle suspension, we imaged the vesicles using confocal microscopy. See Figure 3a for a GUV that was incubated with $3.3 \mu \mathrm{g} / \mathrm{mg}$ of avidin-coated particles, which clearly adhered to the biotincontaining membranes.

As the surrounding liquid is stationary, a diffusion-limited adsorption model seems applicable. ${ }^{44}$ To explain the dependence of particle adsorption on the avidin density, we presume that the avidin concentration on the particles determines the binding probability to biotin-containing membranes. For low membrane coverage, the covered membrane area fraction is proportional to the particle concentration in the surrounding liquid, and therefore, it is reasonable to define a (nonequilibrium) binding affinity with the membrane area that is covered by particles $\phi_{\mathrm{A}}$ divided by the bulk particle volume fraction $\phi_{\mathrm{V}}$ at a fixed incubation time. We measured this membrane area fraction $\phi_{\mathrm{A}}$ using previously described tracking methods on the three-dimensional confocal images. ${ }^{13,34,45}$ The observed binding affinities $\left(\phi_{\mathrm{A}} / \phi_{\mathrm{V}}\right)$ after $30 \mathrm{~min}$ incubation are shown in Figure $3 \mathrm{~b}$. The effect of avidin on the binding affinity to biotin-containing GUVs is clear: between 0 and $0.3 \mu \mathrm{g} / \mathrm{mg}$, the binding affinity increased more than 10 -fold from $1.5 \pm 0.7$ to $18 \pm 4$. After that, the binding affinity increased for increasing avidin densities until a membrane area coverage of $1.9 \%$ after $30 \mathrm{~min}$ in a $0.02 \mathrm{vol} \%$ particle suspension was achieved. At amounts of added avidin larger than $3 \mu \mathrm{g} / \mathrm{mg}$, we observed fluctuations in the binding affinities. Next to random error due to uncertainty in the particle volume fraction, a possible reason for this is the distribution of the avidin proteins over the particles (see Figure 2). From this we conclude that the binding probability of avidin-coated particles increases with increasing avidin density on the particles.

Binding Specificity. We have shown that, by immobilizing a protein on the surface of colloidal particles, the affinity to its target molecule is transferred from the protein to the particle surface. Now, we investigate whether the binding specificity is also conserved. For this, we assessed the binding affinity of three types of functionalized particles to different lipid membranes. As a control experiment, we first tested the binding affinity of particles with only mPEG on their surface. Then, to show the specific targeting to biotinylated surfaces, we used the avidin-coated particles that we investigated in detail in the previous sections ( $3.3 \mu \mathrm{g}$ avidin per mg particles). Finally, to show the generality of the coating protocol, we also assessed particles functionalized with $33 \mu \mathrm{g} / \mathrm{mg}$ of the sugar-binding protein Concanavalin A (ConA).

The specific binding affinities of these three types of particles were first tested on model lipid membranes, GUVs, of known composition (see Experimental Section). In Figure $4 a-c$, we show that none of the investigated particles bound to lipid membranes without exposed biotin molecules: the dense mPEG coating clearly suppressed aspecific binding to the GUVs irrespective of the immobilized proteins that are also present on the particles. On biotinylated membranes, however, we observed binding of avidin-coated particles with high affinity, whereas the control and ConA-coated particles did not show significant binding (Figure $4 d-f$ ). Therefore, we conclude that the coating protocol described here successfully transfers the specificity of the avidin to colloidal particles.

Additionally, we also investigated the specificity of these particles to living cells. For D. discoideum cells, we found that 


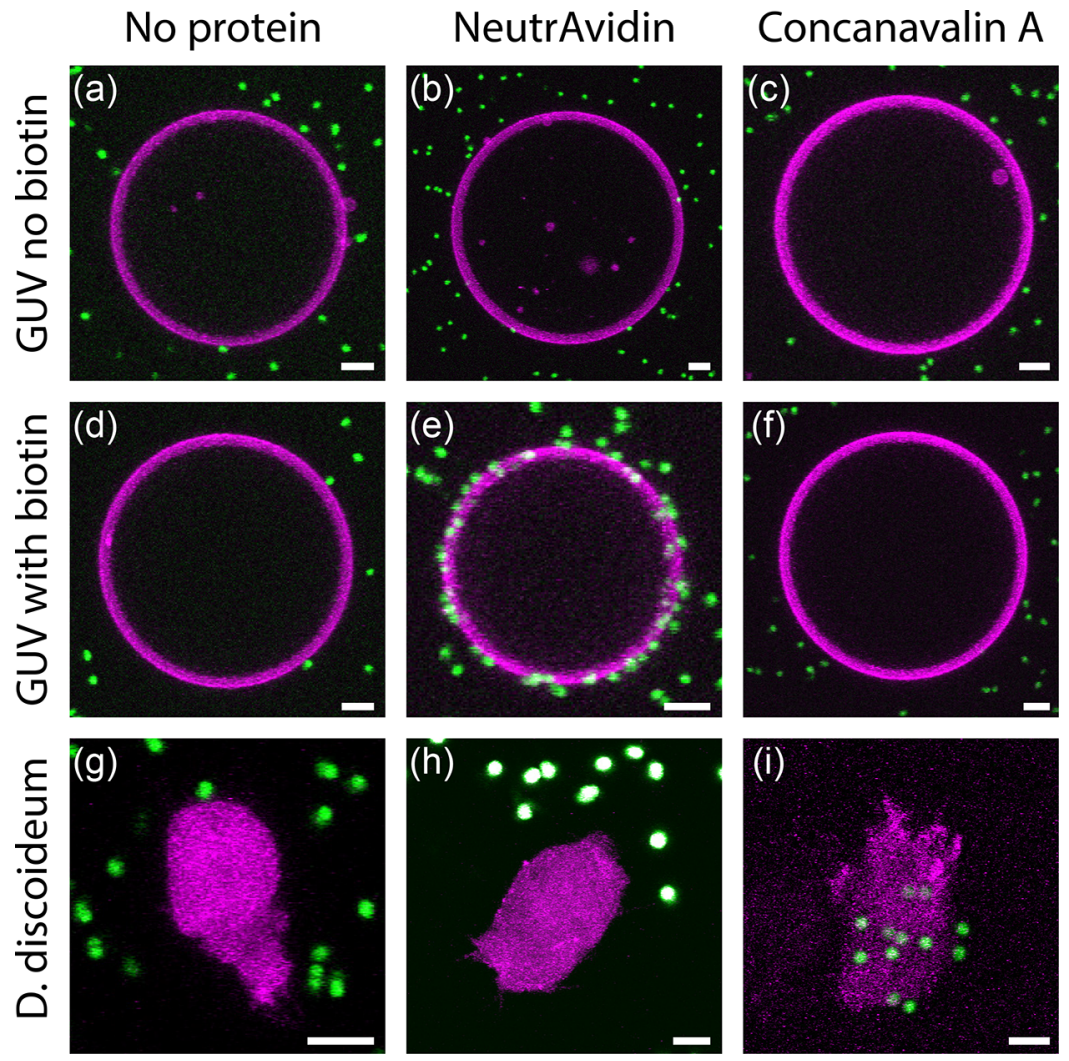

Figure 4. Binding specificity of nonfunctionalized particles, NeutrAvidin-coated particles, and Concanavalin A-coated particles. The specific adhesion is shown on $(a-c)$ GUVs with only PEG on their surface, $(d-f)$ GUVs with an excess of biotinylated mPEG on their surface, and ( $g-i)$ D. discoideum cells in phosphate buffer. All images are maximum intensity projections of three-dimensional confocal images. For the GUVs in (a-f), the particles are displayed in green, and the membrane is displayed in magenta. For the cells in ( $\mathrm{g}-\mathrm{i})$, magenta denotes the filamentous actin, which visualizes the cell contours. These pictures were chosen such that there were no particles floating in front or behind the cells; otherwise, the cells are representative of the samples. The scale bars denote $5 \mu \mathrm{m}$.

the mPEG coating successfully prohibited adhesion to the cells: without $\mathrm{mPEG}$ coating, $50 \%$ of the particles were internalized by the cells, whereas with $\mathrm{mPEG}$ coating, we observed none out of over 200 observed particles to be stuck or internalized (see Figure $4 \mathrm{~g}$ ). On the other hand, on two types of mammalian cells (A549 human lung carcinoma and 3T3 mouse fibroblasts), we found that the mPEG-coated particles stuck nonspecifically to the cellular membranes. As these cells are cultured in protein-rich media, we presume that the observed adhesion was caused by a protein corona that formed around the particles, which is supported by the $\zeta$-potential measurements inside these media (see Table 1). Therefore, we conclude that protein-rich media may induce aspecific binding of the mPEGcoated particles.

The particles that were functionalized with avidin also did not bind to the D. discoideum cells (Figure $4 \mathrm{~h}$ ). These results are in line with the previous results on GUVs: the presence of the immobilized protein does not lead to aspecific binding of the particles. On the other hand, ConA-coated particles did show significant binding to the cells (Figure 4i). ConA binds selectively to glucose and mannose residues ${ }^{46}$ that are present in the glycocortex of the D. discoideum cells. This ConA particle-coating is also specific, which we confirmed by the observation that they did not bind to any of the GUVs (Figure $4 \mathrm{c}$ and $\mathrm{f}$ ). As with the avidin-coated particles to biotinylated membranes, the binding affinity of the ConA particles to the $D$. discoideum cells seems to depend on the amount of ConA on the particles (see Figure S3). Furthermore, we observed that, after cleaving glycoproteins from the cellular membranes by the addition of Trypsin, ${ }^{47}$ the binding affinity decreased significantly.

These observations confirm that the surfactant-free coating protocol described here readily transfers the specificity of a certain protein to a colloidal particle. By suppressing nonspecific binding, our method provides a means to design colloidal particles that "target" specific molecules in lipid membranes, which has useful applications in fundamental membrane studies, micrometer-sized self-assembly, and targeted drug release. We demonstrated this target specificity on GUVs without biotin, GUVs with biotin, and on D. discoideum cells.

\section{SUMMARY AND CONCLUSIONS}

To direct colloidal particles to specific molecules in lipid membranes, we have developed a surfactant-free coating procedure that produces particles with a high affinity to specific molecules. A coating with proteins provides the binding affinity, while at the same time a dense layer of covalently coupled mPEG gives stability at physiological conditions. As the method is surfactant-free, the particles are especially useful for surfactant-sensitive applications, such as studying lipid membrane properties, also in biological environments.

The protein density on the particles can be tuned to adapt the binding affinity, which we have shown for the biotinbinding protein NeutrAvidin using a combined titration and fluorescence assay. The yield of the coating method is such that 
$18 \%$ of the biotin binding sites are accessible after the coating procedure, which is presumably caused by the finite chemical yield, the random protein orientations, and the high mPEG coverage that potentially obstructs the binding sites. The largest observed number of biotin binding sites was $2.9 \times 10^{4}$ per 1.06 $\mu \mathrm{m}$ diameter particle.

These avidin-coated particles spontaneously bind to biotinylated membranes. We have shown that the amount of NeutrAvidin on these particles directly influences the binding affinity. Although particles without avidin did not exhibit any significant binding to these membranes, avidin-coated particles reached up to $1.9 \%$ membrane coverage after $0.5 \mathrm{~h}$ in a 0.02 vol $\%$ particle suspension.

We have shown that the coating procedure can be readily transferred to the sugar-binding protein Concanavalin A. Particles functionalized with this protein become specific to the outside of $D$. discoideum cells, whereas they do not bind to biotinylated membranes. On the other hand, although the avidin-coated particles bind to biotinylated GUVs, they do not bind to these cells. This shows that the specificity of a protein can be transferred successfully to the surface of the colloidal particles.

Our surfactant-free coating method makes colloidal polystyrene particles selectively adhesive to certain targeted molecules at physiological conditions. The fact that the coating procedure only applies covalently linked $\mathrm{mPEG}$ as stabilizer makes it applicable to cases in which surfactants are undesirable. Provided that they remain in their functional state throughout the process, the procedure is readily adjustable to include different proteins. This will provide custom colloidal particles for use in future lipid membrane measurements, micrometer-scale self-assembly, drug targeting, and biosensing applications.

\section{ASSOCIATED CONTENT}

\section{S Supporting Information}

The Supporting Information is available free of charge on the ACS Publications website at DOI: 10.1021/acs.langmuir.7b02065.

Supporting methods and Materials supporting the fluorescence and titration assays (PDF)

\section{AUTHOR INFORMATION}

\section{Corresponding Author}

*E-mail: kraft@physics.leidenuniv.nl.

\section{ORCID}

Casper van der Wel: 0000-0002-0488-2237

Daniela J. Kraft: 0000-0002-2221-6473

\section{Notes}

The authors declare no competing financial interest.

\section{ACKNOWLEDGMENTS}

This work was supported by The Netherlands Organisation for Scientific Research (NWO/OCW) as part of the Frontiers of Nanoscience program and VENI grant 680-47-431.

\section{REFERENCES}

(1) Nagasaki, Y.; Kobayashi, H.; Katsuyama, Y.; Jomura, T.; Sakura, T. Enhanced immunoresponse of antibody/mixed-PEG co-immobilized surface construction of high-performance immunomagnetic ELISA system. J. Colloid Interface Sci. 2007, 309, 524-530.
(2) Kamimura, M.; Miyamoto, D.; Saito, Y.; Soga, K.; Nagasaki, Y. Design of poly(ethylene glycol)/streptavidin coimmobilized upconversion nanophosphors and their application to fluorescence biolabeling. Langmuir 2008, 24, 8864-8870.

(3) Vonarbourg, A.; Passirani, C.; Saulnier, P.; Benoit, J. P. Parameters influencing the stealthiness of colloidal drug delivery systems. Biomaterials 2006, 27, 4356-4373.

(4) Nel, A. E.; Mädler, L.; Velegol, D.; Xia, T.; Hoek, E. M. V.; Somasundaran, P.; Klaessig, F.; Castranova, V.; Thompson, M. Understanding biophysicochemical interactions at the nano-bio interface. Nat. Mater. 2009, 8, 543-557.

(5) Jain, A.; Cheng, K. The principles and applications of avidinbased nanoparticles in drug delivery and diagnosis. J. Controlled Release 2017, 245, 27-40.

(6) Dai, J.; Sheetz, M. P. Mechanical properties of neuronal growth cone membranes studied by tether formation with laser optical tweezers. Biophys. J. 1995, 68, 988-996.

(7) Koster, G.; Cacciuto, A.; Derényi, I.; Frenkel, D.; Dogterom, M. Force barriers for membrane tube formation. Phys. Rev. Lett. 2005, 94, 068101.

(8) Dimova, R.; Dietrich, C.; Hadjiisky, A.; Danov, K.; Pouligny, B. Falling ball viscosimetry of giant vesicle membranes: Finite-size effects. Eur. Phys. J. B 1999, 12, 589-598.

(9) Hormel, T. T.; Kurihara, S. Q.; Brennan, M. K.; Wozniak, M. C.; Parthasarathy, R. Measuring lipid membrane viscosity using rotational and translational probe diffusion. Phys. Rev. Lett. 2014, 112, 188101.

(10) van der Wel, C.; Heinrich, D.; Kraft, D. J. Microparticle assembly pathways on lipid membranes. Biophys. J. 2017, 113, 10371046.

(11) Koltover, I.; Rädler, J. O.; Safinya, C. R. Membrane mediated attraction and ordered aggregation of colloidal particles bound to giant phospholipid vesicles. Phys. Rev. Lett. 1999, 82, 1991-1994.

(12) Sarfati, R.; Dufresne, E. R. Long-range attraction of particles adhered to lipid vesicles. Phys. Rev. E 2016, 94, 012604.

(13) van der Wel, C.; Vahid, A.; Šarić, A.; Idema, T.; Heinrich, D.; Kraft, D. J. Lipid membrane-mediated attractions between curvature inducing objects. Sci. Rep. 2016, 6, 32825.

(14) Li, N.; Sharifi-Mood, N.; Tu, F.; Lee, D.; Radhakrishnan, R.; Baumgart, T.; Stebe, K. J. Curvature-driven migration of colloids on tense lipid bilayers. Langmuir 2017, 33, 600-610.

(15) van der Meulen, S. A. J.; Leunissen, M. E. Solid colloids with surface-mobile DNA linkers. J. Am. Chem. Soc. 2013, 135, 1512915134.

(16) Angioletti-Uberti, S.; Varilly, P.; Mognetti, B. M.; Frenkel, D. Mobile linkers on DNA-coated colloids: valency without patches. Phys. Rev. Lett. 2014, 113, 128303.

(17) van der Meulen, S. A. J.; Helms, G.; Dogterom, M. Solid colloids with surface-mobile linkers. J. Phys.: Condens. Matter 2015, 27, 233101.

(18) Chakraborty, I.; Meester, V.; van der Wel, C.; Kraft, D. J. Colloidal joints with designed motion range and tunable joint flexibility. Nanoscale 2017, 9, 7814-7821.

(19) Lauer, S. A.; Nolan, J. P. Development and characterization of Ni-NTA-bearing microspheres. Cytometry 2002, 48, 136-145.

(20) Wattendorf, U.; Merkle, H. P. PEGylation as a tool for the biomedical engineering of surface modified microparticles. J. Pharm. Sci. 2008, 97, 4655-4669.

(21) Macfarlane, R. J.; Lee, B.; Jones, M. R.; Harris, N.; Schatz, G. C.; Mirkin, C. A. Nanoparticle superlattice engineering with DNA. Science 2011, 334, 204-208.

(22) Wang, Y.; Wang, Y.; Zheng, X.; Ducrot, É; Yodh, J. S.; Weck, M.; Pine, D. J. Crystallization of DNA-coated colloids. Nat. Commun. 2015, 6, 7253.

(23) Wu, G.; Majewski, J.; Ege, C.; Kjaer, K.; Weygand, M. J.; Lee, K. Y. C. Lipid corralling and poloxamer squeeze-out in membranes. Phys. Rev. Lett. 2004, 93, 028101.

(24) Demina, T.; Grozdova, I.; Krylova, O.; Zhirnov, A.; Istratov, V.; Frey, H.; Kautz, H.; Melik-Nubarov, N. Relationship between the structure of amphiphilic copolymers and their ability to disturb lipid bilayers. Biochemistry 2005, 44, 4042-4054. 
(25) Wang, J.-Y.; Chin, J.; Marks, J. D.; Lee, K. Y. C. Effects of PEOPPO-PEO triblock copolymers on phospholipid membrane integrity under osmotic stress. Langmuir 2010, 26, 12953.

(26) Nawaz, S.; Redhead, M.; Mantovani, G.; Alexander, C.; Bosquillon, C.; Carbone, P. Interactions of PEO-PPO-PEO block copolymers with lipid membranes: a computational and experimental study linking membrane lysis with polymer structure. Soft Matter 2012, 8,6744 .

(27) Rusmini, F.; Zhong, Z.; Feijen, J. Protein immobilization strategies for protein biochips. Biomacromolecules 2007, 8, 1775-1789.

(28) Coad, B. R.; Scholz, T.; Vasilev, K.; Hayball, J. D.; Short, R. D.; Griesser, H. J. Functionality of proteins bound to plasma polymer surfaces. ACS Appl. Mater. Interfaces 2012, 4, 2455-2463.

(29) Gopinath, S. C. B.; Tang, T.-H.; Citartan, M.; Chen, Y.; Lakshmipriya, T. Current aspects in immunosensors. Biosens. Bioelectron. 2014, 57, 292-302.

(30) Meng, F.; Engbers, G. H. M.; Feijen, J. Polyethylene glycolgrafted polystyrene particles. J. Biomed. Mater. Res. 2004, 70A, 49-58. (31) Appel, J.; Akerboom, S.; Fokkink, R. G.; Sprakel, J. Facile onestep synthesis of monodisperse micron-sized latex particles with highly carboxylated surfaces. Macromol. Rapid Commun. 2013, 34, 12841288.

(32) Cuatrecasas, P.; Parikh, I. Absorbents for affinity chromatography. Use of N-hydroxysuccinimide esters of agarose. Biochemistry 1972, 11, 2291-2299.

(33) Kada, G.; Falk, H.; Gruber, H. J. Accurate measurement of avidin and streptavidin in crude biofluids with a new, optimized biotinfluorescein conjugate. Biochim. Biophys. Acta, Gen. Subj. 1999, 1427, $33-43$.

(34) Allan, D. B.; Caswell, T. A.; Keim, N. C.; van der Wel, C. Trackpy v0.3.1, Zenodo; 2016, 55143, DOI: 10.5281/zenodo.55143.

(35) Angelova, M. I.; Dimitrov, D. S. Liposome electroformation. Faraday Discuss. Chem. Soc. 1986, 81, 303-311.

(36) de Gennes, P. G. Polymers at an interface; a simplified view. Adv. Colloid Interface Sci. 1987, 27, 189-209.

(37) Upadhyayula, S.; Quinata, T.; Bishop, S.; Gupta, S.; Johnson, N. R.; Bahmani, B.; Bozhilov, K.; Stubbs, J.; Jreij, P.; Nallagatla, P.; Vullev, V. I. Coatings of polyethylene glycol for suppressing adhesion between solid microspheres and flat surfaces. Langmuir 2012, 28, 5059-5069.

(38) Gref, R.; Lück, M.; Quellec, P.; Marchand, M.; Dellacherie, E.; Harnisch, S.; Blunk, T.; Müller, R. H. 'Stealth' corona-core nanoparticles surface modified by polyethylene glycol (PEG): influences of the corona (PEG chain length and surface density) and of the core composition on phagocytic uptake and plasma protein adsorption. Colloids Surf., B 2000, 18, 301-313.

(39) Pelaz, B.; del Pino, P.; Maffre, P.; Hartmann, R.; Gallego, M.; Rivera-Fernández, S.; de la Fuente, J. M.; Nienhaus, G. U.; Parak, W. J. Surface functionalization of nanoparticles with polyethylene glycol: effects on protein adsorption and cellular uptake. ACS Nano 2015, 9, 6996-7008.

(40) Moy, V. T.; Florin, E.-L.; Gaub, H. E. Intermolecular forces and energies between ligands and receptors. Science 1994, 266, 257-259.

(41) de Wolf, F. A.; Brett, G. M. Ligand-binding proteins: their potential for application in systems for controlled delivery and uptake of ligands. Pharmacol. Rev. 2000, 52, 207-236.

(42) Sapsford, K. E.; Algar, W. R; Berti, L.; Gemmill, K. B.; Casey, B. J.; Oh, E.; Stewart, M. H.; Medintz, I. L. Functionalizing nanoparticles with biological molecules: developing chemistries that facilitate nanotechnology. Chem. Rev. 2013, 113, 1904-2074.

(43) Huang, S.-C.; Swerdlow, H.; Caldwell, K. D. Binding the biotinylated DNA to streptavidin-coated polystyrene latex. Anal. Biochem. 1994, 222, 441-449.

(44) Gray, J. J.; Bonnecaze, R. T. Adsorption of colloidal particles by Brownian dynamics simulation: kinetics and surface structures. $J$. Chem. Phys. 2001, 114, 1366-1381.

(45) van der Wel, C. Circletracking v1.0, Zenodo; 2016, 47216, DOI: 10.5281 /zenodo.47216.
(46) Saleemuddin, M.; Husain, Q. Concanavalin A: a useful ligand for glycoenzyme immobilization-a review. Enzyme Microb. Technol. 1991, 13, 290-295.

(47) Phillips, D. R. Effect of trypsin on the exposed polypeptides and glycoproteins in the human platelet membrane. Biochemistry 1972, 11, $4582-4588$. 